\title{
Análisis de una cadena de suministro de autopartes
}

\section{Analysis of a autopart supply chain}

Fernando Gomez Berrezueta

Marco Vinicio Noroña Merchán

Universidad Internacional del Ecuador, Ecuador

Autor para correspondencia: fegomezbe@uide.edu.ec; manoroname@uide.edu.ec

Fecha de recepción: 15 de agosto de 2018 - Fecha de aceptación: 15 octubre de 2018

Resumen: El presente trabajo consiste en el análisis de la estructura y funcionamiento de una cadena de suministro de autopartes en el sector automotriz, considerando los nuevos esquemas de articulación logística involucrados en los eslabones de la cadena para que funcionen como un sistema homogéneo de producción, cumpla las especificaciones y tiempos requeridos. El suministro de autopartes es considerado principal factor de éxito en la industria automotriz, determinando la calidad del medio ambiente y la productividad, por lo cual se analiza la gestión y administración logística del suministro de las autopartes, considerando algunos factores relevantes del sector automotriz desde el punto de vista de diversos autores que han estudiado el tema y luego identificar los esquemas operativos en el suministro de las autopartes. Se analiza la dinámica del sector automotriz a nivel mundial y se describe las características macroeconómicas de la industria manufacturera y de autopartes en México, a continuación se considera factores de la demanda y estrategias de gestión empleadas por los ensambladores de automóviles, se visualiza la estructura y funciones de los miembros de la cadena de suministro y las prácticas actuales de aprovisionamiento de componentes automotrices y al final se presentan las conclusiones derivadas del estudio realizado.

Palabras Claves: cadena de suministro; autopartes; sector automotriz; logística; esquemas operativos

Abstract: The paper consists of the analysis of the structure and functioning of a supply chain of auto parts in the automotive sector, considering the new logistic articulation schemes involved in the links of the chain so that they function as a homogeneous production system, comply with the specifications and times required. The supply of auto parts is considered the main factor of success in the automotive industry, determining the quality of the environment and productivity, for which the management and logistics administration of the supply of the auto parts is analyzed, considering some relevant factors of the automotive sector from the point of view of various authors who have studied the subject and then identify the operational schemes in the supply of auto parts. It analyzes the dynamics of the automotive sector worldwide and describes the macroeconomic characteristics of the manufacturing industry and auto parts in Mexico, then considers factors of demand and management strategies used by automotive assemblers, visualizes the structure and functions of the members of the supply chain and the current practices of automotive components procurement and in the end the conclusions derived from the study carried out are presented.

Key Words: supply chain; auto parts; automotive sector; logistics; operating schemes 


\section{Introducción}

De las 15 mil partes que aproximadamente componen un coche, un fabricante de automóviles diseña y produce solamente algunas, otras son suministradas por sus proveedores directos. Asimismo, los proveedores directos pueden tercerizar sus actividades, al ser abastecidos por otros subproveedores y así sucesivamente.

Se denomina autopartes a la pieza o conjunto de piezas que intervienen en el armado de un automóvil, y que también se venden por separado.

El modelo de referencia SCOR (Supply Chain Operation Reference) contempla seis procesos integradores principales: planear (Plan), abastecer (Source), fabricar (Make), entregar (Deliver), devolver (Return) y habilitar (Enable) con diferentes herramientas y niveles de análisis (Sánchez, 2017).

En la cadena de suministro participan una red de empresas que realizan una planeación estratégica para la creación del ciclo de producción y servicio automotriz, desde el abastecimiento de materias primas hasta la entrega del producto final (automóvil).

La mayoría de las empresas armadoras en México cuenta con empresas de autopartes que se localizan alrededor de sus plantas de vehículos para cumplir con las exigencias de proveeduría y tiempos de entrega (Aguilar-Pérez, 2015). México ofrece a las armadoras una alta capacidad de manufactura y diseño, así como producción de modelos enfocados a los más altos estándares de calidad, comercializados en los mercados de mayor demanda.

\section{Concepto de la cadena de suministro}

Denominada Supply Chain en inglés, es una red de instalaciones y medios de distribución que tiene por función la obtención y transformación de materiales en productos intermedios y productos terminados y distribución de estos productos terminados a los consumidores (Figura $1)$.

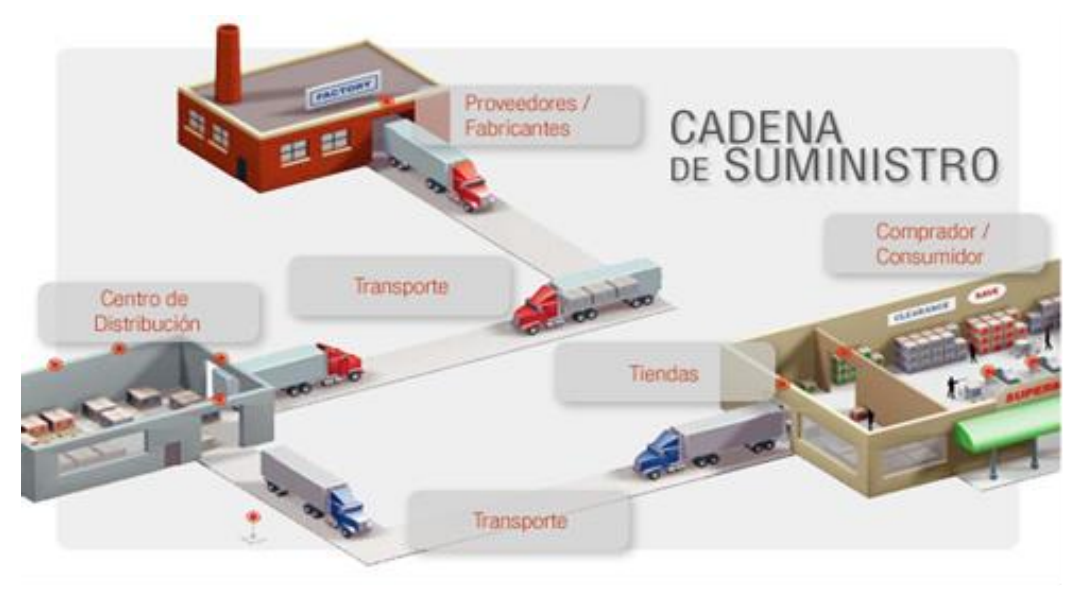

Figura 1. Cadena de suministro (Sintec, 2012)

Según, Michael E. Porter, Ph.D., Harvard University "En el futuro, la competencia no se dará de empresa a empresa, sino más bien de cadena de suministros a cadena de suministros." 
Una cadena de suministro consta de tres partes

1) El suministro: es dónde y cuándo se consiguen y suministran las materias primas para fabricación.

2) La fabricación: convierte estas materias primas en productos terminados.

3) La distribución: se asegura de que dichos productos finales llegan al consumidor a través de una red de distribuidores, almacenes y comercios minoristas.

\section{Objetivos de la cadena de suministro}

1) Promover un adecuado servicio al consumidor final

2) La entrega de los productos en tiempo, forma y calidad

3) Capacidad de entrega de la variedad de los productos

4) Balance adecuado

\section{Tipos de cadenas de suministros}

La cadena de suministros estratégica es la que decide acerca de la tecnología de la producción, el tamaño de la planta, la selección del producto, la colaboración del producto, la colocación del producto en la planta y la selección del proveedor para las materias primas

La cadena de suministros táctica asimila que la cadena de suministros está dada y se encomienda de decidir la utilización de los recursos específicamente: los proveedores, los centros de depósitos y ventas, a través de un horizonte de planificación.

\section{Funciones de la cadena de suministro}

Las funciones que componen la Cadena de Suministro interna a una empresa de manufactura son:

1) Administración del Portafolio de Productos y Servicios (PPS), que es la oferta que la compañía hace al mercado.

2) Servicio a Clientes (SAC), que es responsable de conectar la necesidad del cliente con la operación interna de la compañía.

3) Control de Producción (CP), se encarga de programar la producción interna y, como consecuencia, dispara la actividad de Abastecimiento de insumos.

4) Abastecimiento (Aba), que se encarga de proveer los insumos necesarios para satisfacer las necesidades de Producción (Materia prima y Materiales) 
5) Abastecimiento (Aba)que se encarga de custodiar insumos y producto terminado (en algunas organizaciones solo producto terminado),

Estas 5 funciones deben funcionar coordinadamente para que la Cadena de Suministro interna (o la Logística interna) sea eficiente y efectiva.

\section{Métodos}

\section{Factores tecnológicos que influyen en la logística}

Los factores tecnológicos que influyen en la logística son:

- Inteligencia de datos para gestionar el transporte.

- Modelos de negocios de alta tecnología.

- Uso de información en tiempo real para supervisar todo el proceso de distribución.

- Exploración de flotas autónomas.

La logística se redefine según estas tres tendencias basadas en la tecnología:

\section{Enfoque omnicanal}

Este término se refiere en la logística a la oferta de servicios de entrega a cualquier hora en cualquier lugar. Para afrontar los posibles problemas de tráfico, en países como Indonesia se ha implementado un sistema de casilleros que permite a las personas recoger sus envíos.

\section{Economía colaborativa}

Las empresas de logística actualmente tienden a funcionar sin camiones ni bodegas entre sus activos, transformándose en plataformas electrónicas que permiten brindar servicios de diversos proveedores poniéndolos en contacto con los consumidores.

\section{Inteligencia de datos}

Al tomar las decisiones, cada vez hay más información disponible gracias a los macrodatos. Esta información, puede ser conseguida y procesada en tiempo real, aplicarse en la optimización de rutas de distribución, obtener tiempos de entrega más cortos, realizar pronósticos de la capacidad de transporte y monitorear el estado de lo que se transporta (Head, 2015).

\section{Autopartes en México}

En México, el sector automotriz representa el 6\% del PIB nacional y el 18\% de la producción de manufactura. Cada año la industria automotriz mexicana crece y según algunas proyecciones la producción alcanzará 4 millones de unidades en 2018 y 5 millones en 2020. El auge de la industria automotriz en México, en especial el crecimiento del sector de autopartes se ha iniciado a partir de la firma del Tratado de Libre Comercio de América del Norte (TLCAN); entre 1997 y 2014 su producción creció $971 \%$ y sus exportaciones 1343\% (Viveros, 2014). 
La industria de autopartes se conforma por 727 empresas a nivel nacional de las cuales, alrededor del 30\% son nacionales, según datos de la Secretaria de Economía (2013).

En México operan cerca de 1,000 empresas de autopartes y que el ingreso de nuevas compañías alcanza un crecimiento del 15\%. En 2017 la industria de autopartes facturó 75,000 millones de dólares (mdd), y que lleva a México a la quinta posición mundial en este rubro (Duràn, 2018).

Las grandes compañías ensambladoras, como General Motors, Daimler Chrysler, Ford, Volkswagen, Nissan y otras, denominadas fabricantes de equipo original por sus siglas en inglés (Original Equipment Manufacturer, OEM), han diseñado estrategias de suministro para conseguir el menor costo de producción en el nivel global. Los nuevos esquemas de articulación logística exigen que todos y cada uno de los eslabones de la cadena funcionen en base a un sistema homogéneo de producción, utilizando partes, piezas e insumos intermedios adquiridos en cualquier lugar del mundo y cumpliendo especificaciones uniformes. Lo más importante es la gestión del suministro, el control de los inventarios y la disponibilidad de los componentes automotrices en el sitio de ensamble.

Las empresas fabricantes de autopartes consideran dos instancias de producción:

- La entrega de autopartes a los ensambladores (clientes) para la fabricación de unidades nuevas (equipo original); y

- La generación de inventarios y venta a las mismas armadoras o a otra agencia económica en los circuitos de distribución (mercado de repuestos).

Existen en México 24 complejos productivos en 14 estados del país, en los que se realizan actividades que van desde el ensamble y blindaje, hasta la fundición y el estampado de vehículos y motores y ha ido en aumento por la presencia productiva de las principales empresas armadoras de vehículos (ligeros y pesados) ver Figura 2, tales como: General Motos, Ford, Chrysler, Volkswagen, Nissan, Honda, BMW, Toyota, Volvo y Mercedes-Benz. En México se producen más de 40 modelos de automóviles y camiones ligeros (Escobedo Torres, 2018).

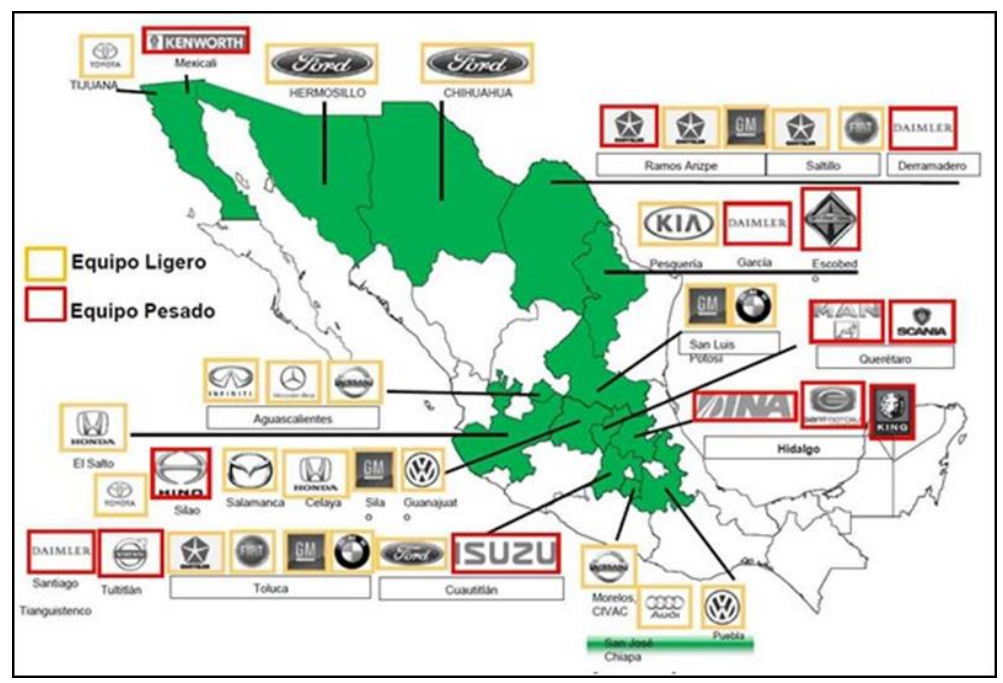

Figura 2. Geografía del Sector Automotor Mexicano (INA, 2018) 
Las empresas que manufacturan autopartes han mejorado sus procesos, debido a la innovación y sofisticación de los productos. Las manufactureras de autopartes se centran en invertir en maquinaria y equipo de punta, así como en la contratación de personal especializado, con la intensión de desarrollar productos innovadores que respondan a las exigencias del mercado (ProMéxico, 2014).

\section{Análisis y desarrollo}

\section{Estructura de la cadena productiva de la industria automotriz}

Es posible identificar que está formada por tres grandes eslabones.

1) Está conformado por la fundición de piezas de metal, suministro de piezas de polímeros,

2) plásticos y diversos materiales.

3) Consta de la fabricación específica de partes, dentro de las cuales existen procesos muy diversos que no siguen un encadenamiento lineal, sino procesos de diferente complejidad. Estos dos eslabones corresponden, en términos generales, al segmento de autopartes.

4) Luego se encuentra el ensamble de los distintos sistemas nodales con que cuenta el vehículo, como: carrocerías, suspensión, motores, sistema eléctrico y frenos, etc. Estos sistemas se integran en el proceso de ensamble del vehículo que finaliza con el producto terminado. Posteriormente está la comercialización del automóvil y el servicio postventa, que se efectúa a través de distribuidores autorizados.

Todas estas partes corresponden a la cadena de valor de la industria automotriz, desde el proceso de estampado y manufactura hasta el servicio posventa (Bohórquez Cruz, 2016).

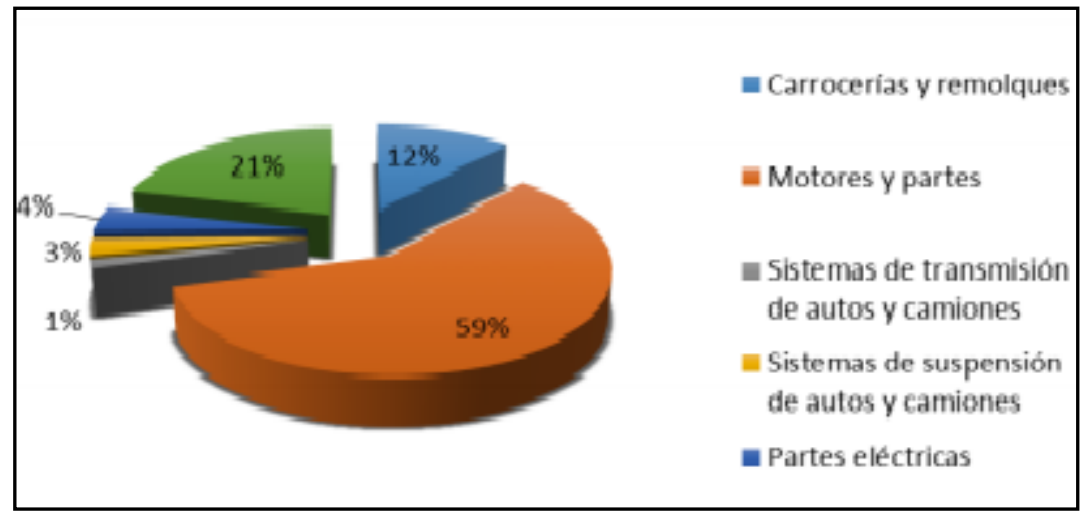

Figura 3. Empresas de autopartes en México (INA, 2018).

Existen tres factores importantes que influyen los cambios en la industria automotriz mundial:

1) el comportamiento de la demanda de vehículos,

2) la regulación gubernamental en los países desarrollados y

3) los cambios tecnológicos

Las ensambladoras se involucran cada vez más en servicios relacionados con el automóvil y ocupan menos en manufactura y ensamblado, encomendando la responsabilidad a 
los proveedores. Este comportamiento está estrechamente relacionado con su lógica de evaluación de desempeño en el que el incremento del valor de las acciones de la empresa es lo más importante (Álvarez Medina, 2002).

\section{Composición del sector de empresas de autopartes}

La producción de automóviles muestra una cadena de actividades productivas que transforman materias primas y autopartes en un vehículo terminado. La cadena contiene actividades de servicios financieros, de publicidad y de distribución para el consumidor final. En el proceso se involucran empresas de varios tamaños y localizadas en diferentes países, ya sea en la producción de autopartes, ensamblaje final o ventas.

La producción de la industria de autopartes se destina principalmente a la industria terminal de automóviles, pero también se destina al mercado de repuestos. El mercado de repuestos se conforma de la forma siguiente:

1. partes o piezas destinadas a la reparación de automóviles chocados $29.9 \%$;

2. piezas de desgaste $21.8 \%$;

3. partes mecánicas $19.5 \%$;

4. equipo y accesorios $15.8 \%$; $y$,

5. piezas de repuesto $14.0 \%$.

En la Figura 4 se observa las posibilidades del mercado de autopartes.

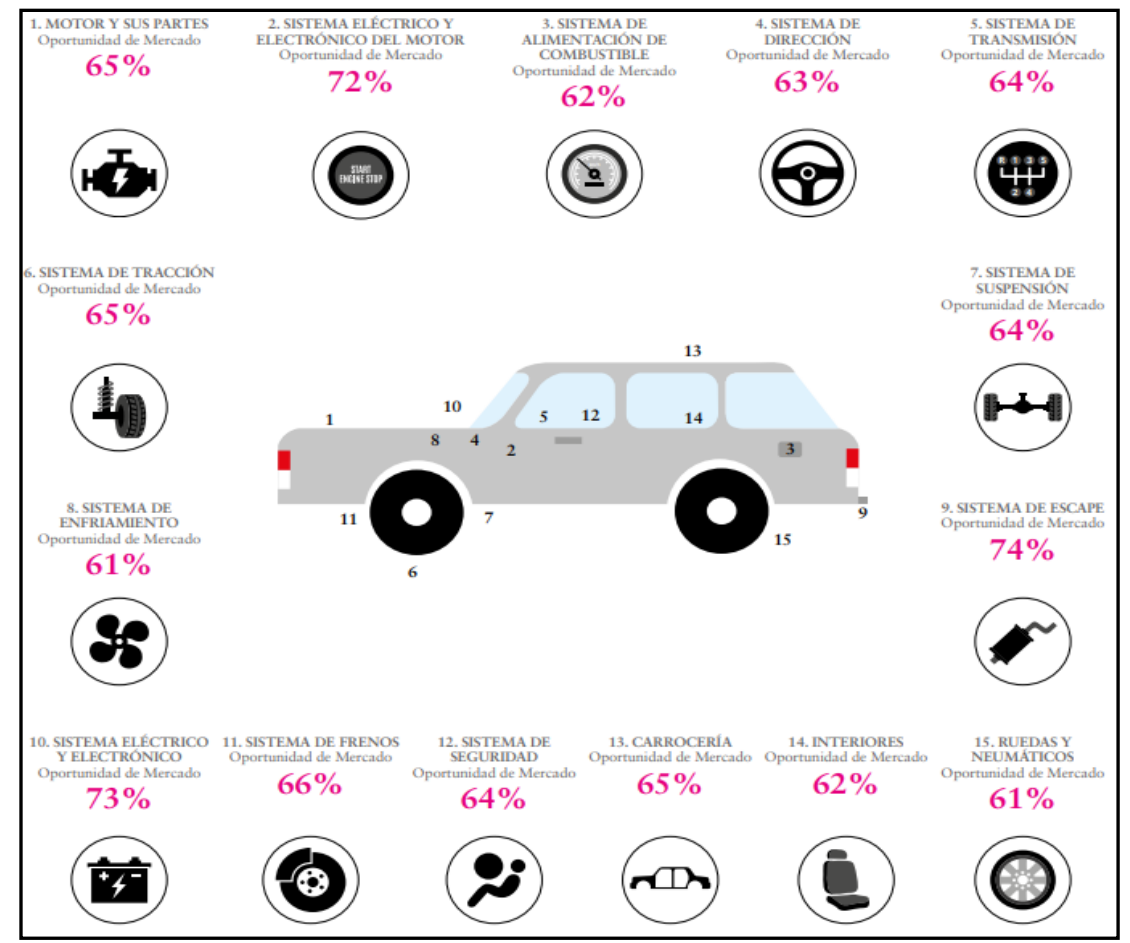

Figura 4. Participación de las oportunidades en el mercado de componentes y sistemas automotrices (ProMéxico, 2016)

\section{Proveedores de la cadena de suministro}


Los proveedores se clasifican en función de su distancia con el OEM (Figura 5).

Tier 1. Proveedores de partes originales (también conocidas como proveedores de primer nivel) a las ensambladoras, principalmente de subensambles y tiene capacidad de diseño.

Tier 2 y 3. Proveedores de partes con diseños suministrados por Tier 1, generalmente suministran productos relativamente básicos y partes individuales. También conocidos como proveedores de segundo y tercer nivel.

De acuerdo con información del Sistema Empresarial Mexicano (SIEM), en territorio nacional se encuentran más de 600 empresas de autopartes, de las cuales aproximadamente el $30 \%$ son de primer nivel (Tier 1). Se considera que alrededor de una tercera parte de las empresas de autopartes son Tier 1

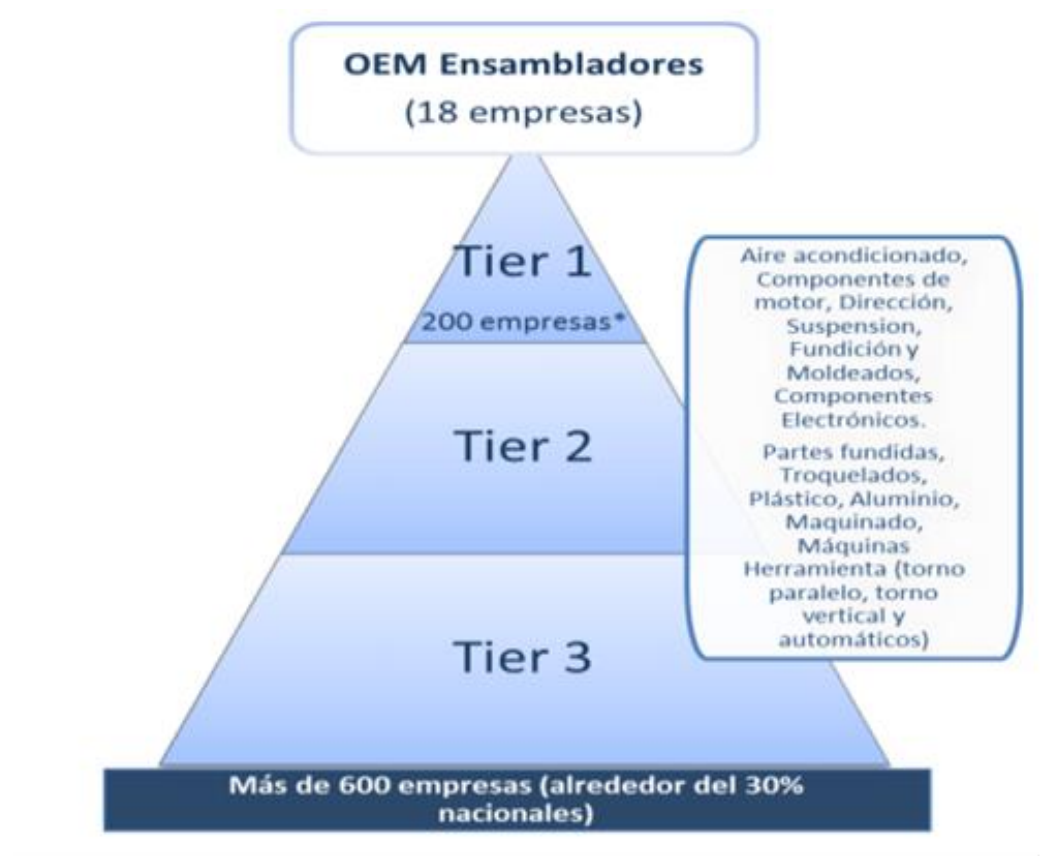

Figura 5. Proveedores de autopartes (SE, 2018)

Las empresas extranjeras Tier 1 contribuyen a fortalecer la posición de México como productor y exportador de autopartes. México presenta áreas de oportunidad en el desarrollo de proveedores mexicanos en los niveles Tier 2 y Tier 3. En este ámbito, ProMéxico ha reconocido oportunidades para el sector automotriz terminal y de autopartes que van orientadas al encadenamiento productivo de ambos sectores. La estrategia se enfoca en proveedores Tier 2 y Tier 3 dedicados al procesamiento de materiales que por su especialización y calidad agregan valor en el producto final; ProMéxico busca atraer al país a empresas extranjeras que contribuyan al desarrollo de esta proveeduría y que, mediante alianzas o coinversiones, transfieran conocimiento y tecnología a las empresas mexicanas. Gráficamente las oportunidades de inversión identificadas por ProMéxico en la cadena de abastecimiento automotriz. 


\section{Resultados}

La propuesta de VW (autos modulares) que consiste en: Estandarizar piezas y procesos, otorgando grandes ventajas a la industria automotriz, en términos de reducir costos y agilizar la producción. Volkswagen apuesta por la armonización de sus plantas en todo el mundo. Se montan las piezas individuales en módulos completos de diversas familias y se asegura la entrega de partes y módulos a las líneas de producción sincronizadamente.

Centros Multi-Clientes para ensambles y secuenciados (Mul- ti JIT-JIS Center): SEGLO logistics desarrolla un modelo de negocio dirigido específicamente para las necesidades que cada día toman más fuerza en la cadena de suministro de la industria automotriz en México: los centros de sub-ensambles y secuenciados. Es una instalación externa localizada en una ubicación estratégica entre el OEM y varios proveedores TIER 1, en donde se elaboran operaciones de secuenciado y sub-ensamble para diversas etapas de la cadena de ensamble del OEM con la finalidad de integrar su cadena de suministro y así consolidar sus costos fijos, laborales y operativos (Figura 6).

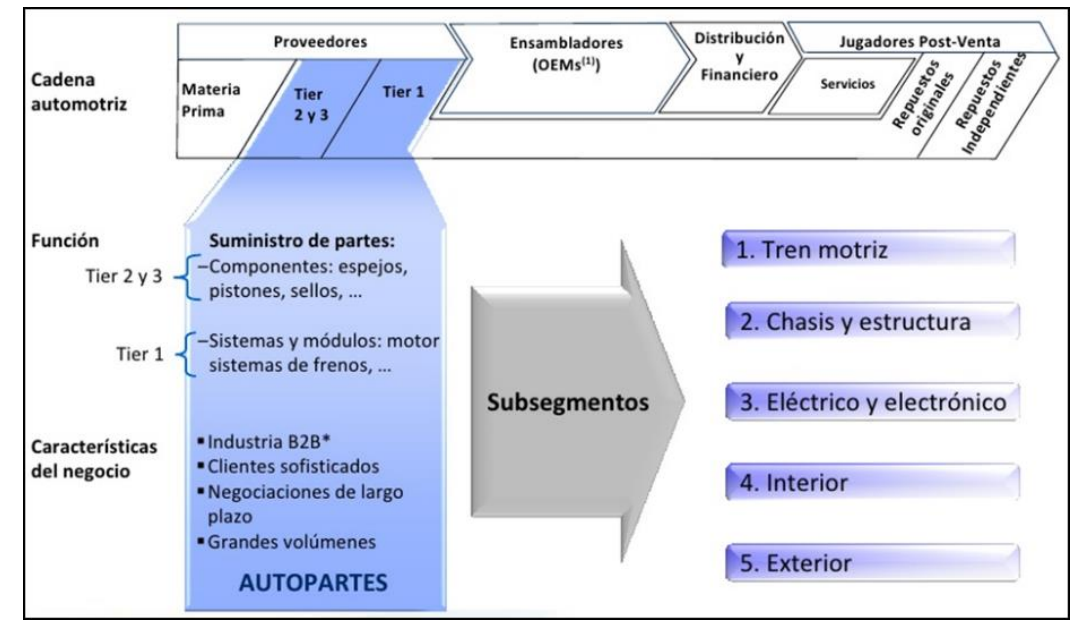

Figura 6. Cadena de suministro automotriz (ProMèxico, 2016)

\section{Procesos involucrados en la cadena de suministro}

Para visualizar el esquema de organización de la cadena de suministro de la industria automotriz en función de la gestión de calidad (Figura 7), partiendo desde las OEM's (Original Equipment Manufacturer: Fabricantes de Equipos Originales), tenemos los siguientes procesos:

1. Planificación

2. Aprovisionamiento

3. Pedidos por emergencia

4. Cumplimiento de pedidos

5. Traslados y despacho

6. Proceso de análisis de existencias

La norma ISO/TS 16949, establece los requisitos para implementar un sistema de gestión que permite cumplir con las especificaciones necesarias para el desarrollo de productos, 
fabricación y en los casos en los que procede, ensamble y mantenimiento del producto y está diseñada para cumplir con los requisitos del sector automotriz. La certificación de dicha normativa aplica sólo al sitio de manufactura.

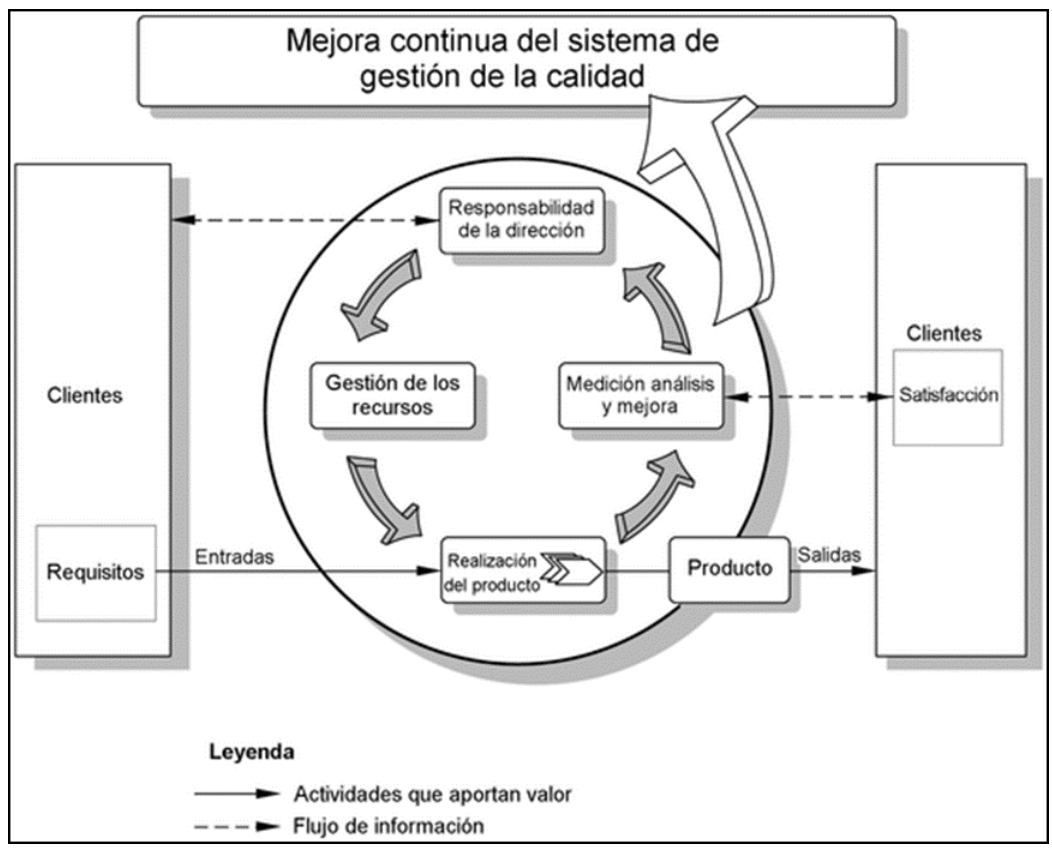

Figura 7. Modelo de un sistema de gestión de la calidad basado en procesos (Norma ISO 9001:2008)

\section{Conclusiones}

Se dice que la cadena comienza con los proveedores de tus proveedores y termina con los clientes de tus clientes.

Todos los proveedores de bienes y servicios y todos los clientes están eslabonados por la demanda de los consumidores de productos terminados, al igual que los intercambios materiales e informáticos en el proceso logístico, desde la adquisición de materias primas hasta la entrega de productos terminados al usuario final.

La revolución en los métodos de producción busca cada vez más flexibilidad que colabore con la disminución de los costos. La competencia de los vehículos y componentes llegados desde países de economías emergentes hace necesaria la reinvención de los métodos de producción.

La Cadena de Suministros contiene aquellas actividades agrupadas con el movimiento de bienes desde el suministro de materias primas hasta el consumidor final.

La logística busca ofrecer un servicio de calidad al entregar productos a los clientes al menor costo y la cadena de suministros quiere asegurarse que todos los costos en la cadena se mantienen controlados para lo que es necesario desde un inicio encontrar las mejores materias primas a un buen precio. 
Se les conoce como TIER 1 a los proveedores directos de las OEM y son estrictamente vigilados en materia de la calidad, tiempo y costos de todos sus componentes y partes de subensambles. En este orden, TIER 2, son las compañías que surten de componentes a las TIER 1 y así sucesivamente con los TIER 3, que proveen a los TIER 2.

\section{Bibliografía}

Aguilar-Pérez, P. \&.-C. (2015). Esquema de condicionantes en la relación proveedor-cliente en la industria automotriz. Caso sector autopartes en la Zona del Bajío. . Dirección y Organización 56, 57-67.

Álvarez Medina, M. (2002). Cambios en la industria automotriz frente a la globalización: el sector de autopartes en México. Contaduría y Administración, (206), 29-49.

Bohórquez Cruz, M. F. (2016). Análisis de los factores de diferenciación del flujo comercial del sector autopartes México-Colombia (2010-2014).

Contreras, O. F. (2006). Redes de producción global y aprendizaje local: El caso de Ford en Hermosillo . V Congreso Nacional de la Asociación Mexicana de Estudios del Trabajo. Oaxtepec, Morelos.

Duràn, A. (Abril de 2018). Sube México en ranking de exportación de autopartes. Obtenido de http://www.elfinanciero.com.mx/empresas/sube-mexico-en-ranking-de-exportacion-deautopartes

Escobedo Torres, C. \&. (2018). El Escenario Global-Local: Nissan En Aguascalientes.

Head, K. (Noviembre de 2015). Word Economic Forum. Obtenido de https://www.weforum.org

INA. (2018). Industria Nacional de Autopartes. Obtenido de https://www.ina.com.mx/

Liker, J. K. (2006). Japanese automakers, US suppliers and supply chain superiority. Supply Chains and Total Product Systems: A Reader.

Moreno, F. M. (2016). Vehículos Eléctricos. Historia, Estado Actual Y Retos Futuros. European Scientific Journal, ESJ, 12(10).

Norma ISO 9001:2008. (2008). Modelo de un sistema de gestión de la calidad basado en procesos.

ProMèxico. (2016). La Industria Automotriz Mexicana: Situación Actual, Retos Y Oportunidades. Mèxico.

Sánchez, M. \&. (2017). Análisis, diagnóstico y propuesta de mejora para una cadena de suministro de autopartes en el Perú utilizando el modelo SCOR.

SE. (2018). Secretaria De Economia. Obtenido de http://www.economia.gob.mx/files/comunidad_negocios/industria_comercio/Monografia _Industria_Automotriz_MARZO_2012.pdf 
Sintec. (2012). http://www.sintec.com/expertise/consultoria-en-cadena-de-suministro/. Obtenido de http://www.sintec.com/expertise/consultoria-en-cadena-de-suministro/

Viveros, J. C. (2014). El auge de la industria automotriz en México en el siglo XXI. Reestructuración y catching up. 